\title{
An unusual cause of gross hematuria: Questions
}

\author{
Aysel Taktak • Banu Acar • Gökçe Gür • Tuğrul Tiryaki • \\ Aynur Albayrak • Nilgün Çakar
}

Received: 23 September 2013 / Revised: 26 September 2013 / Accepted: 3 October 2013 /Published online: 20 December 2013

(C) IPNA 2013

Keywords Child $\cdot$ Hematuria

\section{Case presentation}

An 8-year-old male patient was admitted with intermittent gross hematuria and dysuria of 1 month's duration. Prior to visiting our hospital he had been treated with several antibiotics despite negative urine cultures. He had no history of any allergies, genitourinary problems or external trauma. According to his medical history, he had been diagnosed with autism 2 years previously, and had been treated with valproic acid since then. His family history was insignificant.

There were no pathological findings on physical examination. Macroscopically, the urine was red. Urinalysis revealed hematuria, minimal proteinuria, no pyuria or casts. Urine culture was negative. Spot urine calcium/creatinine ratio was normal (0.04).

The answers to these questions can be found at http://dx.doi.org/10.1007/ s00467-013-2670-x.

A. Taktak $(\triangle) \cdot$ B. Acar $\cdot$ G. Gür $\cdot$ N. Çakar Department of Pediatric Nephrology and Rheumatology, Ankara

Child Health, Hematology, Oncology Education and Research

Hospital, Ankara, Turkey

e-mail: aysel.taktak@gmail.com

T. Tiryaki

Department of Pediatric Surgery, Ankara Child Health, Hematology, Oncology Education and Research Hospital, Ankara, Turkey

\footnotetext{
A. Albayrak

Department of Pathology, Ankara Dışkapı Yıldırım Beyazıt,

Education and Research Hospital, Ankara, Turkey
}

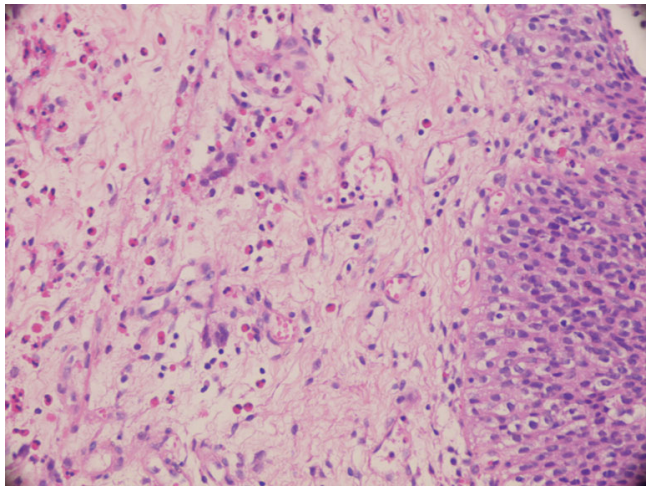

Fig. 1 Infiltration of the bladder mucosa and submucosa by numerous eosinophils

Laboratory studies revealed a white blood cell count of $7,900 / \mathrm{mm}^{3}$ with peripheral eosinophilia $(10 \%)$. The serum creatinine level was $0.42 \mathrm{mg} / \mathrm{dl}$, blood urea nitrogen was $26 \mathrm{mg} / \mathrm{dl}$, electrolytes were normal. Immunological studies showed markedly elevated serum IgE $(1,050 \mathrm{IU} / \mathrm{ml})$. Bladder wall thickness was $8 \mathrm{~mm}$ under ultrasound with no upper urinary tract and renal pathology. The etiology of gross hematuria remained uncertain; thus, further investigation was performed.

Cystoscopy revealed edematous and hyperemic bladder wall and urethra, but no petechia or bleeding. Histopathological examination showed an infiltration of the mucosa and submucosa by numerous eosinophils (Fig. 1).

\section{Questions}

1. What is the most likely diagnosis?

2. What additional diagnostic examinations would you perform?

3. What treatment should be given? 